\title{
How chemical and physical mechanisms enable the influence of the operating conditions in a photocatalytic indoor air treatment device to be modeled
}

\author{
Frédéric Batault ${ }^{\mathrm{a}, \mathrm{b}, \mathrm{c}}$, Valérie Héquet ${ }^{\mathrm{a}}$, Cécile Raillard ${ }^{\mathrm{a}, *}$, Frédéric Thévenet ${ }^{\mathrm{b}, \mathrm{c}}$, Nadine Locoge ${ }^{\mathrm{b}, \mathrm{c}}$, \\ Laurence Le Coq ${ }^{\mathrm{a}}$ \\ ${ }^{a}$ GEPEA, UMR CNRS 6144, Mines Nantes, DSEE, 4 rue Kastler, BP 20722, 44307 Nantes, Cedex 3, France \\ ${ }^{\mathrm{b}}$ Mines Douai, SAGE, F-59508 Douai, France \\ ${ }^{\mathrm{c}}$ Université Lille Nord de France, F-59000 Lille, France
}

Keywords:

Photocatalysis

Indoor air

Experimental design

Reaction rate law

\begin{abstract}
A B S T R A C T
The photocatalytic degradation of toluene in indoor air conditions was performed in a closed-loop multipass photocatalytic reactor using the Box-Behnken experimental design methodology. The objective of this work was to rigorously determine a kinetic model in order to understand the behavior of the reactor in real indoor air conditions and to relate the kinetic parameters to physical and chemical mechanisms. Three main parameters were studied: initial toluene concentration, light irradiance and air stream velocity. The experimental results were used to calculate the single-pass removal efficiency for different operating conditions and to establish a relationship between the single-pass removal efficiency, light irradiance and air stream velocity. This relationship was integrated into an overall reaction rate law based on the Langmuir-Hinshelwood mechanism. The kinetic model obtained was then validated for various experimental conditions.
\end{abstract}

\section{Introduction}

Indoor air quality (IAQ) has become an important issue in industrialized countries due to the time spent by many people in closed environments. Indoor air pollution includes several potentially harmful biological, organic and inorganic species arising from numerous outdoor and indoor sources [1]. Among indoor pollutants, Volatile Organic Compounds (VOCs) represent an ongoing priority for the authorities [2]. Indoor air pollutants are generally present at concentrations ranging from fractions of ppbv to tens of ppbv while the concentration of water vapor can reach about 20,000 ppmv indoors. Poor indoor air quality may cause health disorders such as respiratory health effects [3], drowsiness, headache, sore throat, mental fatigue or, more generally, Sick Building Syndrome (SBS) [4,5]. To avoid or reduce the impact of bad IAQ on human health, the control of pollutant emissions and increased building ventilation rates are proposed. However, these strategies are not always possible or efficient depending on the outdoor environment and may generate additional energy consumption. Thus, indoor air treatment systems appear to be interesting alternative solutions.

Several processes can remove VOCs from indoor air. Advanced Oxidation Processes (AOPs) have received growing attention due to their minimal waste production and low energy consumption.

Photocatalytic oxidation of VOCs is considered a promising AOP for indoor air quality improvement. In the last decade, many studies have been conducted on the photocatalytic oxidation of gaseous VOCs. The total oxidation of VOCs has already been evidenced in batch laboratory reactors [6-8] and several authors have 
successfully adapted the technique for flow reactors with a view to developing indoor air treatment systems further [9-12]. However, several points still need to be more thoroughly investigated to assess and ensure the efficiency of the process and to obtain a tool enabling the scale-up, design and optimization of large-scale photocatalytic systems $[13,14]$. One of these points is the study of the influence of controlled and uncontrolled conditions. Controlled conditions refer to process parameters such as flow rate, irradiance, UV wavelength, etc. Uncontrolled parameters depend on features of the air stream to be treated, such as the type of pollutants, concentrations, and air humidity. In the literature, studies for the development of kinetic models are generally performed at ppmv levels of VOCs [15-19], which are higher than typical indoor air VOC concentrations. It was the case of a previous study performed in the laboratory and already published [20]. The general objectives were to establish a kinetic law, modeling the photocatalytic degradation of three VOCs and taking into account the influence of two controlled parameters - irradiance and upstream velocity - and the initial concentration of pollutants. However in this previous work, the lowest studied concentration was fixed at $5 \mathrm{ppmv}$. The model was built using experimental designs and was validated for different experimental conditions. It was shown that the model fitted the experimental results properly during the first $60 \mathrm{~min}$ of reaction but tended to diverge with time, i.e. for lower VOC concentrations [20]. Furthermore, in this previous work, a polynomial function was used to express the influence of the operating parameters so that mostly mathematical considerations were taken into account while physico-chemical mechanisms needed to be further explored. Thus, the resulting rate law suffered from a drawback: it was only valid for the domain defined by the experimental conditions. Based on that, the remaining issue related to the determination of a kinetic law valid in a more realistic range of concentration and independent of process parameters.

Consequently, the present work deals with the photocatalytic oxidation of toluene, a typical indoor air pollutant and model molecule in numerous studies, in humid air at sub-ppmv concentrations. This study was conducted in a closed-loop reactor and investigated the influence of three operating conditions: initial toluene concentration, light irradiance and flow rate. The two latter are process parameters that can be controlled to optimize the reactor performance [20].

The main objectives of this work were to propose a kinetic model in order to understand the behavior of the reactor in real indoor air conditions and to relate the kinetic parameters to physical and chemical mechanisms. The kinetics of degradation of toluene were obtained using a Box-Behnken experimental design.
In a first part, statistical analysis of results obtained from the DOE were discussed leading to general trends and also highlighting limitations of this approach. In a second part, the calculation of the single-pass removal efficiency (RE) using results from the DOE was proposed. Finally, this alternative approach has permitted the writing of a rate law as a function of the operating parameters.

\section{Experimental methods}

The experimental set-up used in this study is described in Fig. 1. It consists of the photocatalytic reactor, the VOC generation bench and the analytical device.

\subsection{Photocatalytic reactor}

The 420 L-dynamic photocatalytic reactor, operating in a continuous mode, has been described in previous articles [20,21] and was slightly modified for the present study. It is a closedloop duct entirely built in stainless-steel to minimize surface losses of analytes and allowing experiments to be conducted at sub-ppmv concentrations. A variable-speed fan operating inside the reactor is used to recirculate air $\left(20-150 \mathrm{~N} \mathrm{~m}^{3} \cdot \mathrm{h}^{-1}\right)$. The flow rate is determined by measuring the pressure drop between the two sides of a calibrated diaphragm. A honeycomb part enables a homogeneous flow distribution in the tranquilization chamber, where the injection of toluene and sampling for analysis are carried out.

The photocatalytic module includes the photocatalytic medium and the UV lamps. The medium was supplied by the Ahlström Paper Group. It was specifically produced for this work and was used as a pleated filter. It consisted of P25 $\mathrm{TiO}_{2}$ deposited on organic fibers (cellulose, polyester and polyamide). $\mathrm{TiO}_{2}$ particles were mixed with a silica binder to ensure a good grip on the woven support. The $\mathrm{TiO}_{2}$ load was 17 g.m ${ }^{-2}$. The medium was $250 \mu \mathrm{m}$ thick. Its overall BET specific surface area, measured by a Micromeritics ASAP 2010 device, was $31 \pm 1 \mathrm{~m}^{2} . \mathrm{g}^{-1}$. A pleated geometry was used to increase the photocatalytic medium surface area to $1100 \mathrm{~cm}^{2}$ and thus the mass of $\mathrm{TiO}_{2}$ used to $1.87 \mathrm{~g}$. Fig. 2 illustrates the configuration of the PCO filter and gives the spectrum of the lamps used in this study. The medium was irradiated with 18-W UVA fluorescent tubes (Philips PL-L series) placed inside the filter folds as shown in Fig. 2. The same photocatalytic media was reused for every experiment. Between two experiments, the photocatalytic media was cleaned by flushing humid VOC-free air with the UV lamps switched on for at least $8 \mathrm{~h}$. A control experiment was carried out at regular intervals to make sure that the photocatalyst was not deactivated.

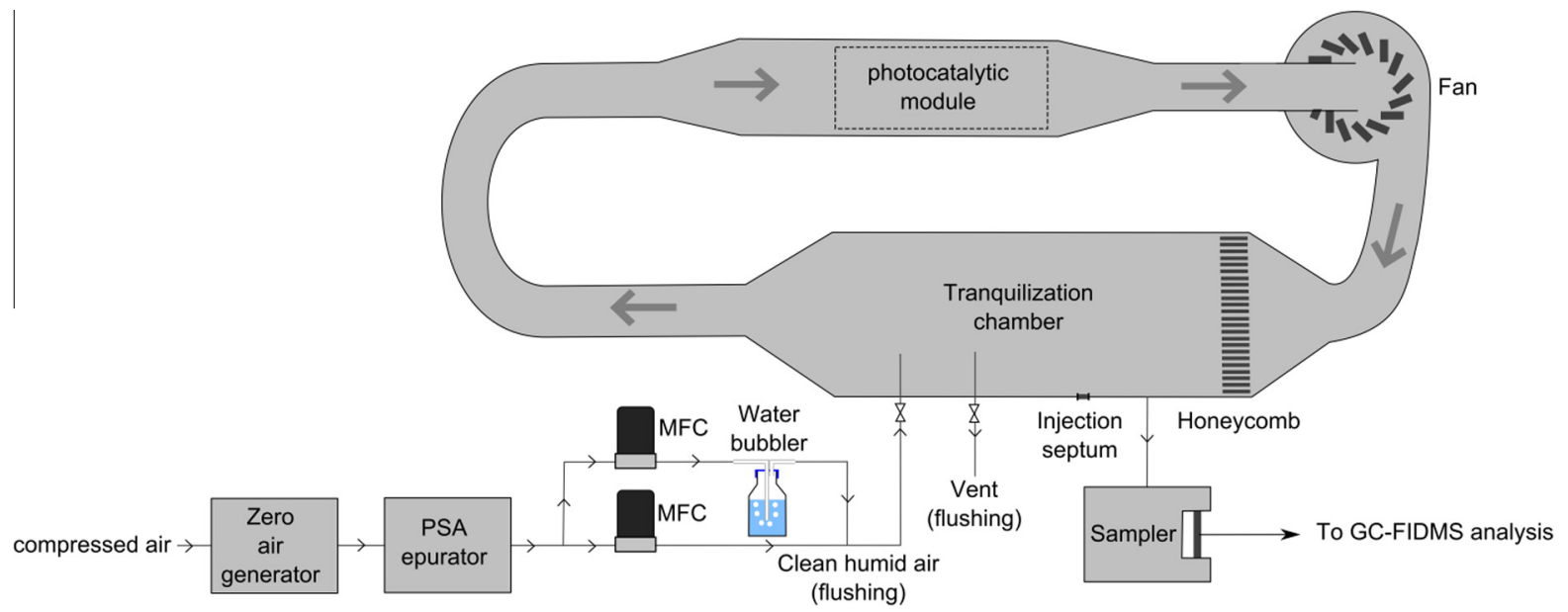

Fig. 1. Dynamic photocatalytic reactor and experimental devices for VOC generation and analysis. 

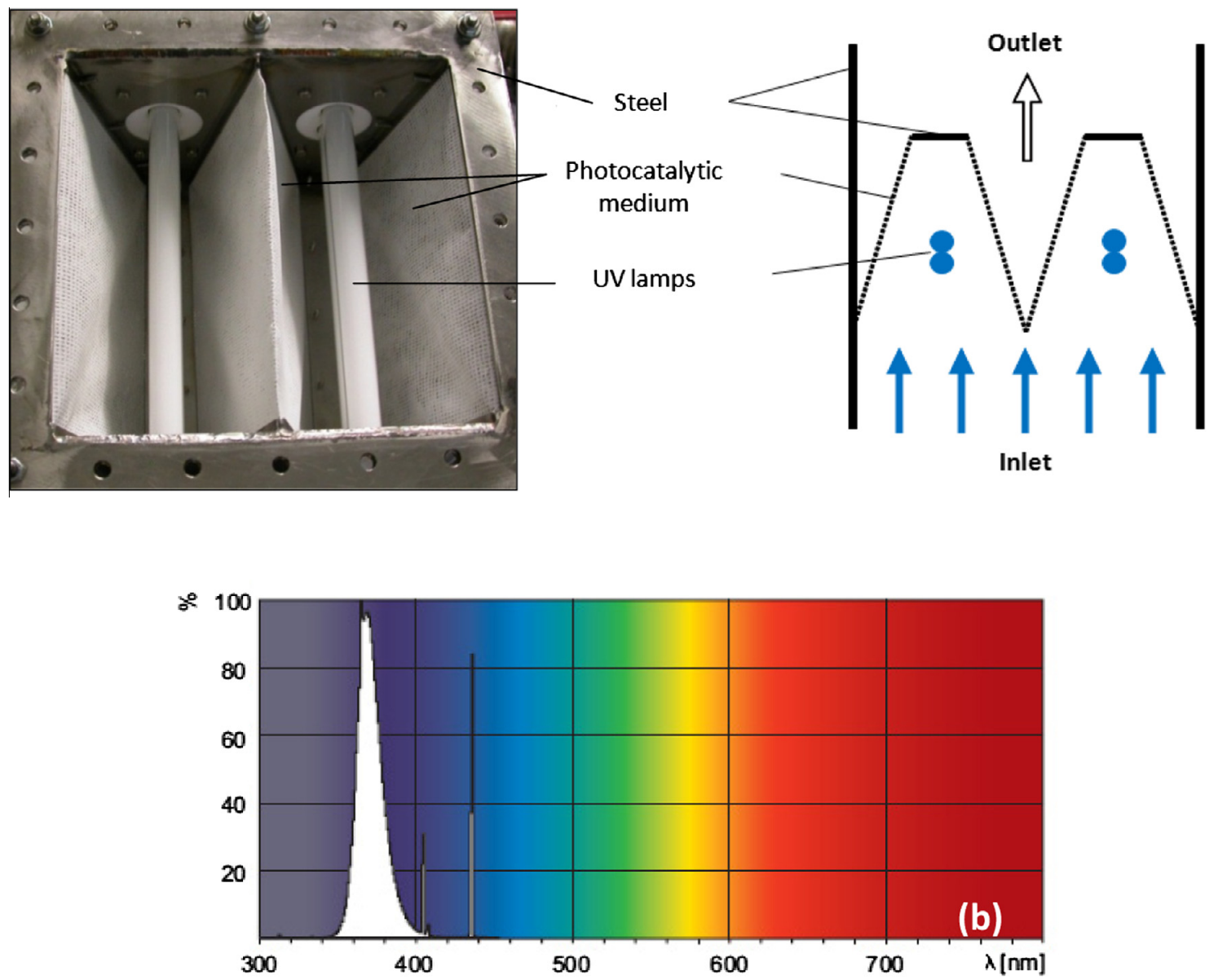

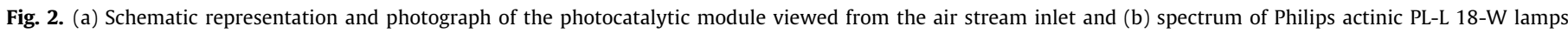
(Philips data).

A variable voltage supply was used to modify the light intensity provided by the lamps and consequently the irradiance received by the photocatalytic medium. Before the experiments, the average light irradiance on the photocatalyst was determined for different voltages applied to the lamps and air velocities. Actually the air velocity has an indirect influence on the light irradiance because the air flow slightly modifies the temperature of the lamps. For this purpose, the light irradiance was first measured for a single voltage at several positions of the photocatalyst with a Vilber Laurmat VLX $3 \mathrm{~W}$ radiometer equipped with a calibrated $C X-365$ sensor. Then to obtain a more accurate distribution of the light irradiance and to better define the average light irradiance for all set voltage supply, it was chosen to complete with a theoretical method of calculation of the light irradiance; the Taranto et al. method [22]. The obtained light distribution is given in Fig. 3. For this setting (15\% of the nominal voltage), the irradiance on the photocatalytic surface ranged between 0 and $0.9 \mathrm{~mW} . \mathrm{cm}^{-2}$ and the average value was $0.35 \mathrm{~mW} . \mathrm{cm}^{-2}$. The measured and calculated irradiances were compared for validation and calibration. All these steps enabled the average light irradiance to be controlled during the experiments by knowing the required lamp voltage for any desired average irradiance on the photocatalyst surface and any air flow velocity.

\subsection{VOC generation and analytical methods}

Liquid toluene (99.99\% purity, purchased from VWR) was evaporated in a 1 -L glass reactor at room temperature to prepare the target gas phase concentrations. Gaseous toluene was then injected into the tranquilization chamber of the photocatalytic pilot by means of a $50-\mathrm{mL}$ syringe through a septum. The concentration of toluene in the photocatalytic reactor was monitored during the experiments by sampling on Perkin-Elmer sorbent cartridges. These cartridges contained three beds of Perkin Elmer activated carbon: Carbopack B, Carbopack C and Carbopack X. Sampling was done with a Markes FLEC Air Pump 1001 sampler at room temperature and a $100 \mathrm{~mL} / \mathrm{min}$ flow. Sorbent tubes were analyzed by thermal desorption/gas chromatography/flame ionization detector and mass spectrometry (TD/GC/FID/MS Perkin-Elmer). A 60-m Rxi-624Sil MS non-polar column was used with TD desorption at $250{ }^{\circ} \mathrm{C}$ and trapping at $-30^{\circ} \mathrm{C}$. The flame ionization detector was used for quantification of toluene and was coupled to a mass spectrometer operated in electron impact mode for chromatographic peak identification. The calibration curve for toluene was performed by diluting a standard toluene cylinder (1040 ppbv in $\mathrm{N}_{2}$, Prax'air) with clean air. The detection limit was estimated to be lower than 21 ppbv.

\subsection{Reactor preparation}

Before each experiment, the reactor was flushed with VOC-free and humidity-controlled air to remove traces of VOCs and to establish the desired humidity in the reactor. The quality of air entering the reactor was obtained using the following preparation: compressed air was treated with a zero air generator Claind 2020, then humidity and $\mathrm{CO}_{2}$ were removed using a suppressor TDGSi PSA 


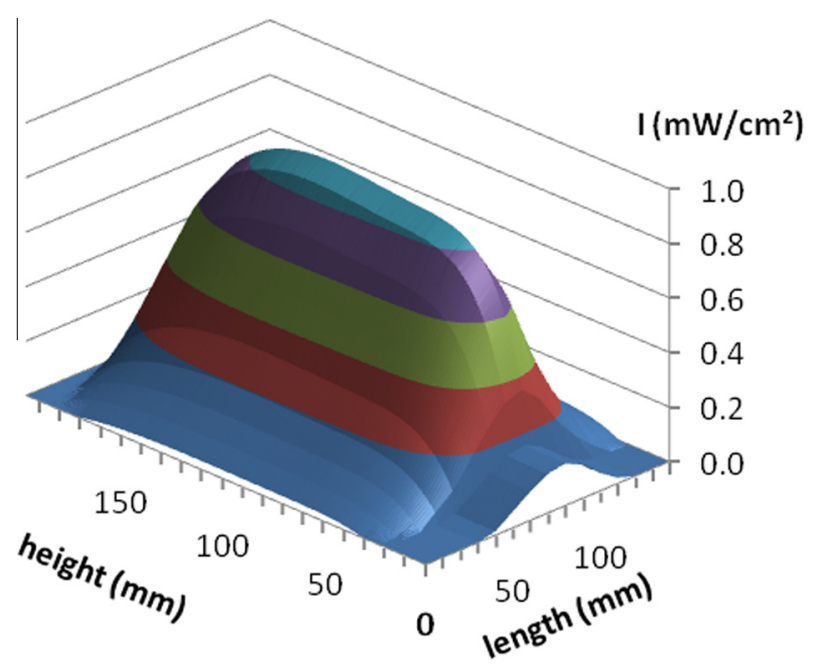

Fig. 3. Light distribution on one side of the photocatalytic surface for $15 \%$ of the nominal voltage supplied to the lamps.

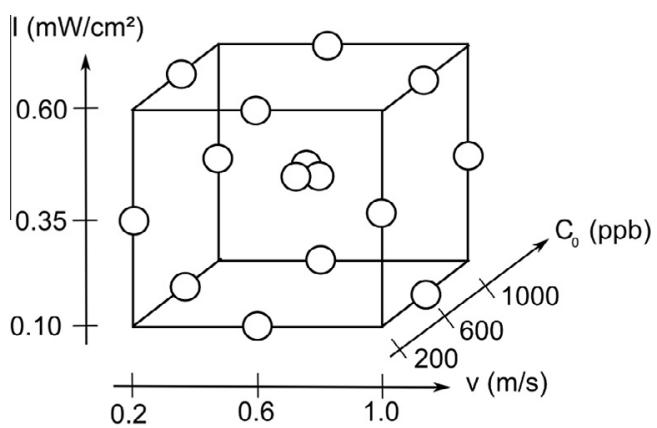

Fig. 4. Experimental conditions for each parameter studied in the framework of the Box-Behnken design of experiments.

device, and finally the air stream was re-humidified to $50 \%$ relative humidity (hygrotransmitter measurement) with a room temperature bubbler and according to an appropriate gas dilution using two Brooks mass flow controllers. About $15 \mathrm{ppm}$ of $\mathrm{CO}_{2}$ remained in the pilot before the start of an experiment.

\subsection{Experimental strategy}

The influence of three operating parameters was investigated by means of a design of experiments (DOE). The first parameter was light irradiance $I\left(\mathrm{~mW} . \mathrm{cm}^{-2}\right)$. It was controlled by the lamp voltage, as explained above, and ranged from 0.1 to $0.6 \mathrm{~mW}$. $\mathrm{cm}^{-2}$. The second parameter was the air flow rate passing through the photocatalytic module in the reactor, expressed as the stream velocity $v\left(\mathrm{~m} . \mathrm{s}^{-1}\right)$. It ranged from 0.2 to $1 \mathrm{~m} . \mathrm{s}^{-1}( \pm 1 \%)$ and was calculated by dividing the air flow rate by the $0.04 \mathrm{~m}^{2}$ section $(20 \mathrm{~cm} \times 20 \mathrm{~cm}$ square-section pipe), Reynolds number over 2500. The third parameter was the initial toluene concentration $C_{0}$. Three levels were used for each parameter (given in Fig. 4), for a better assessment of the non-linear variations. To decrease the number of experiments, the fractional Box-Behnken design was chosen [23]. In this design, the experimental conditions were distributed as shown in Fig. 4. The initial toluene concentration ranged between $200 \mathrm{ppbv}\left(0.76 \mathrm{mg} \cdot \mathrm{m}^{-3}\right)$ and $1000 \mathrm{ppbv}$ (3.79 mg.m $\mathrm{m}^{-3}$ ). Twelve sets of experimental conditions corresponded to the middle points of the cube edges. One corresponded to the central point of the cube. Three experiments were performed with the central conditions to assess the random variations of the response. These fifteen experiments were carried out in a random order.

\section{Results and discussion}

The influence of the studied parameters may be assessed by means of several kinds of experimental responses. In this study, the degradation rate was chosen as the response to analyze the results. To obtain the degradation rate of toluene, kinetic curves were plotted, analyzed and decomposed in order to determine the relevant kinetic constants.

\subsection{General analysis of degradation kinetic curves}

The objective of this part was to find appropriate kinetic descriptors that would then be used to establish a reaction rate law as a function of the three studied parameters. This was done by plotting the concentration of toluene, or the logarithm of the concentration, versus time. Fig. 5a gives an example of the curve for the following experimental conditions: $\mathrm{I}=0.35 \mathrm{~mW} . \mathrm{cm}^{-2}$, $\mathrm{v}=0.2 \mathrm{~m} . \mathrm{s}^{-1}, \mathrm{C}_{0}=200 \mathrm{ppbv}$. This plot indicates that the kinetics cannot be fitted with a first-order model. A LangmuirHinshelwood-like model (Eq. (1)) was thus preferred, which is widely used for photocatalytic reactions $[24,25]$. It is easily demonstrated that this model tends to first-order kinetics for low concentrations. This behavior is observed on the curve plotted in Fig. $5 \mathrm{a}$.

$\frac{d C}{d t}=\frac{k \cdot K \cdot C}{1+K \cdot C}$

To model the experimental kinetic curves, the analytical solution of Eq. (1) was calculated. The resulting relation is given by Eq. (2). The experimental curves were thus fitted with this relation and it was found that the proposed model fitted the experimental data properly for all the performed experiments $\left(R^{2}=0.969\right.$ for the experimental conditions in Fig. 5). For instance, Fig. 5b shows the fit using the Langmuir-Hinshelwood model for the same experimental conditions as in Fig. 5a.

$t=A \cdot\left[B \cdot\left(C-C_{0}\right)+\ln \frac{C}{C_{0}}\right]$

Eq. (2) expresses the relation between the concentration $C$ (ppbv) and the time $t(\mathrm{~h})$ during the photocatalytic degradation of toluene. This equation involves three constants: the initial concentration $C_{0}$ (ppbv), $A(\mathrm{~h})$ and $B\left(\mathrm{ppbv}^{-1}\right)$. The curve fit provided the values of $A, B$ and $C_{0}$ for each experiment. These were used to recalculate two other kinetic descriptors: the initial reaction rate $r_{0}\left(\mathrm{ppbv} \cdot \mathrm{h}^{-1}\right.$ ) and the apparent first-order kinetic constant for the late part of the curves $k^{\prime}\left(\mathrm{h}^{-1}\right)$. These descriptors were calculated with Eqs. (3) and (4) below and the results are presented in Table 1.

$r_{0}=\frac{-1}{A} \cdot \frac{C_{0}}{1+B \cdot C_{0}}$

$k^{\prime}=\frac{-1}{A}$

In the following part, the influence of the parameters $I, v$ and $C_{0}$ on the kinetic descriptors $r_{0}$ and $k^{\prime}$ is investigated.

\subsection{Analysis of data obtained from the design of experiments}

A statistical analysis was used to assess the influence of $I, v$ and $C_{0}$ on $r_{0}$ and $k^{\prime}$. This data analysis was performed with the Statgraphics Centurion software. It is based on the fit of an output number $y$ as a function of several variables $x_{i}$ with a quadratic multi-variable function, which uses ten $a_{i}$ and $a_{i j}$ constants. This 

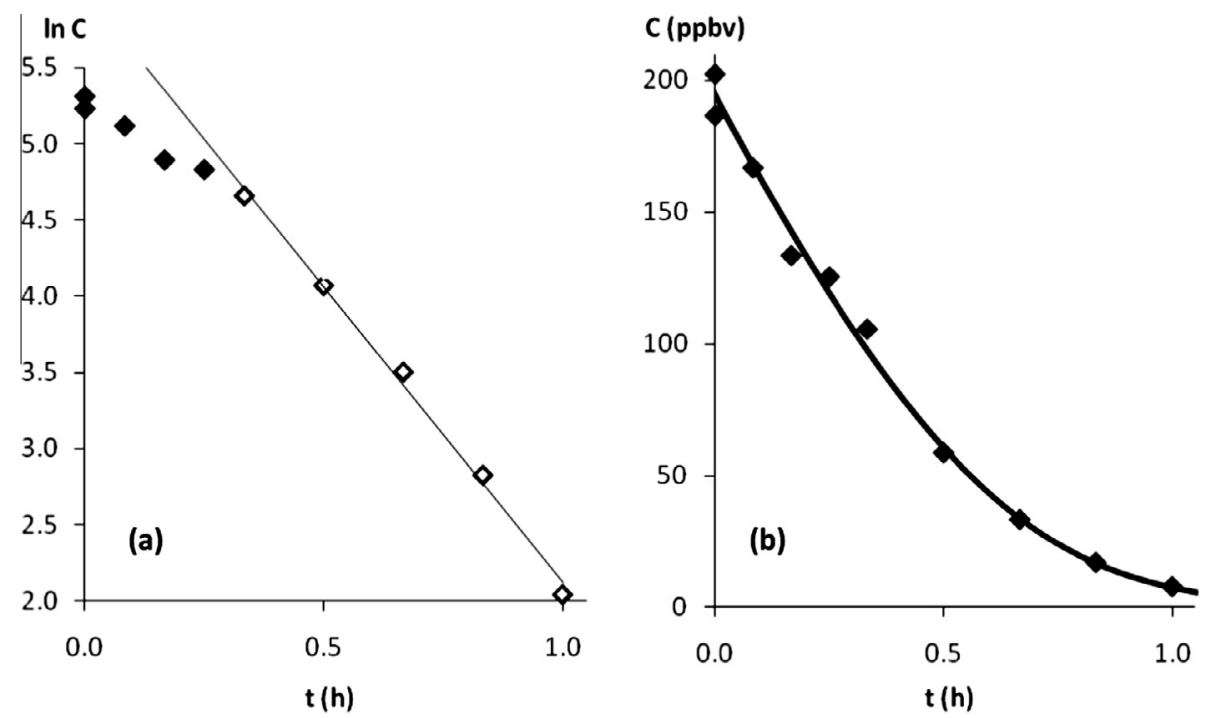

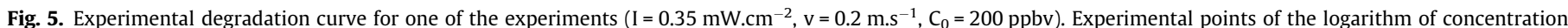

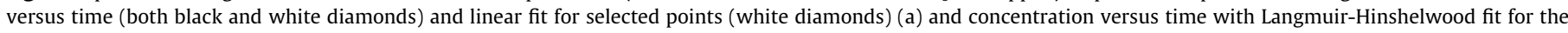
whole experimental points (b).

Table 1

Calculated values of the kinetic descriptors $A, B, C_{0}, k^{\prime}$ and $r_{0}$ for the 15 experiments of the DOE.

\begin{tabular}{|c|c|c|c|c|c|c|c|}
\hline \multicolumn{3}{|c|}{ DOE operating parameters } & \multicolumn{5}{|c|}{ Calculated kinetic descriptors } \\
\hline$I\left(\mathrm{~mW} \cdot \mathrm{cm}^{-2}\right)$ & $v\left(\mathrm{~m} \cdot \mathrm{s}^{-1}\right)$ & $C_{0}(\mathrm{ppbv})$ & $A(\mathrm{~h})$ & $B \cdot 10^{5}\left(\mathrm{ppbv}^{-1}\right)$ & $C_{0}(\mathrm{ppbv})$ & $k^{\prime}\left(\mathrm{h}^{-1}\right)$ & $r_{0}\left(\mathrm{ppbv}_{\mathrm{h}} \mathrm{h}^{-1}\right)$ \\
\hline 0.35 & 0.6 & 600 & -0.153 & 241 & 656 & 6.54 & 1662 \\
\hline 0.10 & 0.2 & 600 & -0.361 & 52 & 692 & 2.77 & 1411 \\
\hline 0.60 & 0.6 & 1000 & -0.111 & 195 & 1018 & 9.01 & 3072 \\
\hline 0.60 & 1.0 & 600 & -0.154 & 145 & 503 & 6.49 & 1890 \\
\hline 0.60 & 0.2 & 600 & -0.161 & 124 & 610 & 6.21 & 2154 \\
\hline 0.10 & 1.0 & 600 & -0.340 & 191 & 581 & 2.94 & 809 \\
\hline 0.35 & 1.0 & 200 & -0.117 & 1040 & 180 & 8.55 & 534 \\
\hline 0.60 & 0.6 & 200 & -0.131 & 235 & 234 & 7.63 & 3964 \\
\hline 0.35 & 0.6 & 600 & -0.142 & 276 & 647 & 7.04 & 1636 \\
\hline 0.35 & 0.2 & 1000 & -0.222 & 189 & 1183 & 4.50 & 1646 \\
\hline 0.10 & 0.6 & 1000 & -0.352 & 350 & 1049 & 2.84 & 638 \\
\hline 0.10 & 0.6 & 200 & -0.228 & 1530 & 198 & 4.39 & 215 \\
\hline 0.35 & 0.2 & 200 & -0.178 & 1120 & 222 & 5.62 & 357 \\
\hline 0.35 & 1.0 & 600 & -0.149 & 367 & 799 & 6.71 & 1363 \\
\hline 0.35 & 0.6 & 600 & -0.165 & 128 & 706 & 6.06 & 2251 \\
\hline
\end{tabular}

function enables the calculation of nine effects from three parameters: three 1-parameter linear effects, three 1-parameter nonlinear effects and three 2-parameter bilinear cross effects. The statistical calculation indicates the significance level of each of the nine effects. This significance is expressed as p-values. For each effect, the p-value corresponds to the probability that the observed $y$ variation when changing the $x_{i}$ parameter(s) is mere coincidence instead of being due to the $x_{i}$ variation. Here, $y$ is either $r_{0}$ or $k^{\prime}$ calculated from the 15 experiments and $x_{i}$ are $I, v$ and $C_{0}$ (Eq. (5)). The methodology was applied separately for each kinetic descriptor $r_{0}$ and $k^{\prime}$.

$$
\begin{aligned}
y= & a_{0}+a_{1} \cdot I+a_{2} \cdot v+a_{3} \cdot C_{0}+a_{11} \cdot I^{2}+a_{12} \cdot I \cdot v+a_{13} \cdot I \cdot C_{0} \\
& +a_{22} \cdot v^{2}+a_{23} \cdot v \cdot C_{0}+a_{33} \cdot C_{0}^{2}
\end{aligned}
$$

Table 2 indicates the p-values for the $a_{i}$ and $a_{i j}$ coefficients of Eq. (5) calculated by the Student test ( $t$-test) considering $r_{0}$ and $k$ ' as DOE responses. The $\mathrm{p}$-value is given in percentage. If the $\mathrm{p}$-value is lower than $5 \%$, it means that the factor or the interaction is significant. Otherwise, i.e. if the p-value is higher than $10 \%$, the effect of the factor or interaction is due to mere coincidence.

It can be seen in Table 2 that for both $r_{0}$ and $k^{\prime}$, only light irradiance $I$ shows a significant effect as the p-values are $2.3 \%$ and
Table 2

p-values in percentage obtained for $r_{0}, k^{\prime}$ and $R E$ (removal efficiency), significant effects are in bold.

\begin{tabular}{llll}
\hline Coefficient & $\mathrm{r}_{0}$ & $\mathrm{k}^{\prime}$ & $\mathrm{RE}$ \\
\hline$a_{1}$ & $\mathbf{2 . 3}$ & $\mathbf{0 . 2 5}$ & $\mathbf{0 . 0 2 0}$ \\
$a_{2}$ & 56 & 20 & $\mathbf{0 . 0 0 0}$ \\
$a_{3}$ & 80 & 47 & 39 \\
$a_{11}$ & 43 & 9.0 & $\mathbf{3 . 6}$ \\
$a_{12}$ & 95 & 85 & $\mathbf{0 . 5 6}$ \\
$a_{13}$ & 54 & 23 & 16 \\
$a_{22}$ & 17 & 20 & $\mathbf{0 . 1 1}$ \\
$a_{23}$ & 54 & 90 & 12 \\
$a_{33}$ & 45 & 65 & 19 \\
\hline
\end{tabular}

$0.25 \%$, respectively. It can be deduced from these results that Eq. (5) does not satisfactorily describe the effect of the three operating parameters $I, v$ and $C_{0}$ on $r_{0}$ and $k^{\prime}$. The statistical approach to assess the dependency of $r_{0}$ and $k^{\prime}$ on $I, v$ and $C_{0}$ was not sufficient enough in this case.

Thus, one solution was to propose a semi-empirical rate law by taking into account the main physical and chemical phenomena occurring during the photocatalytic degradation. To establish this 
Table 3

Obtained values of kinetic descriptors $A$ and $B$ for the three experiments with the DOE central point experimental conditions $\left(C_{0}=600 \mathrm{ppbv}, I=0.35 \mathrm{~mW} . \mathrm{cm}^{-2}, v=0.6 \mathrm{~m}\right.$. $\left.\mathrm{s}^{-1}\right)$.

\begin{tabular}{llll}
\hline & $A(\mathrm{~h})$ & $\begin{array}{l}B \cdot 10^{-5} \\
\left(\mathrm{ppbv}^{-1}\right)\end{array}$ & $\begin{array}{l}C_{0} \\
(\mathrm{ppbv})\end{array}$ \\
\hline Trial 1 & -0.153 & 241 & 656 \\
Trial 2 & -0.142 & 276 & 647 \\
Trial 3 & -0.165 & 128 & 706 \\
Average and standard & $-0.153 \pm 0.012$ & $215 \pm 77$ & $667 \pm 31$ \\
$\quad$ deviation & & & \\
\hline
\end{tabular}

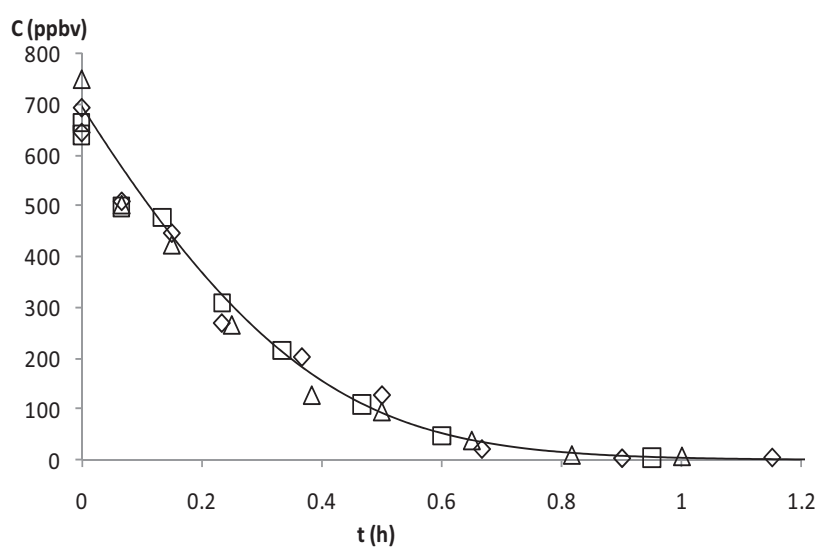

Fig. 6. Degradation curves for the three experiments with the DOE central point experimental conditions $\left(C_{0}=600 \mathrm{ppbv}, I=0.35 \mathrm{~mW} . \mathrm{cm}^{-2}, v=0.6 \mathrm{~m} . \mathrm{s}^{-1}\right)$.

law, attention was focused on the influence of the three parameters $I, v$ and $C_{0}$ on the descriptor $B$ first. Then in a second step, instead of descriptor $A$, which is equivalent to $\mathrm{k}^{\prime}$, another kinetic descriptor was introduced in order to distinguish the different effects of stream velocity $v$ on the overall reaction rate. This descriptor is the single-pass removal efficiency of toluene, called $R E$. The influence of the studied operating parameters on the $R E$ descriptor was also calculated for the further writing of the reaction rate law.

\subsection{Influence of the operating parameters on the kinetic descriptor $B$}

For the 15 experiments carried out in the conditions fixed by the DOE, the kinetic descriptor $B$ was calculated according to the methodology described in Section 3.1. The obtained values ranged from $52 \cdot 10^{-5} \mathrm{ppbv}^{-1}$ to $1530 \cdot 10^{-5} \mathrm{ppbv}^{-1}$ and showed large variations; no significant correlations with the operating parameters $I, v$ and $C_{0}$ were found (Table 1 ). Furthermore, even for identical experimental conditions, the calculation of the kinetic descriptor B gave very different results (Table 3). Fig. 6 gives the degradation curves of three experiments carried out in identical experimental conditions (central point of the DOE: $C_{0}=600 \mathrm{ppbv}$, $\left.I=0.35 \mathrm{mw} . \mathrm{cm}^{-2}, v=0.6 \mathrm{~m} . \mathrm{s}^{-1}\right)$. It can be noticed that the three degradation curves overlap well, indicating that the results are repeatable. The three curves were then fitted with the methodology presented above and the kinetic descriptors $A, B, r_{0}$ and $k$ ' were calculated for the three trials. The values obtained from these calculations are summarized in Table 3. The relative error on the calculation of $B$ reached $36 \%$ while it was only $7.5 \%$ for the kinetic descriptor $A$ and $4.5 \%$ for the initial concentration $C_{0}$. These experiments evidenced that the obtained $B$ values can be very different for the same shape of curve, indicating that the determination of $B$ by curve fitting is very sensitive. The observed variations in $B$ between these three experiments were comparable to those observed in the full set of experiments, indicating that they were due to the curve fitting rather than the effects of the operating conditions. The determination of $\mathrm{B}$ is not robust enough using the described methodology.

In order to write a reaction rate law involving the main operating parameters, and particularly to take into account the effect of air velocity $v$, it was necessary to examine another kinetic descriptor i.e. to focus on the effect of $I$ and $v$ on the single-pass removal efficiency $(R E)$.

\subsection{Determination of the single-pass removal efficiency $R E$}

To study the effect of air velocity $v$, it should be noted that several effects related to this parameter may co-exist. For example, the rate of reactions occurring in the photocatalytic module depends on both the amount of molecules entering this module per unit of time (mass flow rate) and the proportion of these molecules being transformed while flowing through the module (single-pass efficiency). Both categories of effects depend on the air velocity parameter. The mass flow rate effect depends on the reactor configuration, closed-loop as in the present study or a single-pass reactor. Its contribution is positive and linear. The second effect, the single-pass efficiency, is intrinsic to the photocatalytic module whatever the reactor configuration. It is controlled by several air-velocity-dependent physical phenomena like mass transfer and contact time. To assess the effect of air velocity on the degradation rate better, it was necessary to calculate the single-pass removal efficiency for different experimental conditions. This enabled the influence of the mass flow rate to be distinguished from the intrinsic efficiency of the photocatalytic module.

The removal efficiency of a photocatalytic system is generally determined by comparing the inlet and outlet concentrations, $C_{\mathrm{IN}}$ and $\mathrm{C}_{\text {Out. }}$. It is defined as the ratio between the consumed and the introduced molecules (Eq. (6)). The reaction rate can be written as the product of the number of molecules entering the reactor per time unit and the removal efficiency (RE). The number of molecules entering the reactor per unit of time is given by the air flow rate $Q_{V}$ and the inlet concentration $C_{I N}$. The reaction rate $r$ is thus given by Eq. (7).

$R E=\frac{C_{I N}-C_{\text {OUT }}}{C_{I N}}$

$r=C_{I N} \cdot Q_{v} \cdot R E$

For a closed-loop reactor in a multi-pass mode, the same kind of calculation can be done. In this case, the aim is to calculate the removal efficiency $R E$ (\%), knowing the overall reactor reaction rate $r$ (ppbv. $\mathrm{h}^{-1}$ ). For this purpose, the reaction rate at the photocatalytic module inlet can be written as a variation in concentration $d C_{I N}$ (ppbv) during a time $d t(\mathrm{~h})$, as shown in Eq. (8).

$r=\frac{-d C_{I N}}{d t}$

For further calculations, the period $d t$ is considered equal to the time $T$ needed for a molecule to complete a whole round of the closed-loop reactor. This duration can be used as a differential time if it is short in comparison with the characteristic degradation time. $T$ is calculated by dividing the reactor volume $V\left(\mathrm{~m}^{3}\right)$ by the flow rate $Q_{V}\left(\mathrm{~N} \mathrm{~m}^{3} \cdot \mathrm{h}^{-1}\right)$, as written in Eq. (9). This hypothesis was experimentally verified: the mixing time of the reactor was much larger than the one-round time $T$. The value of $T$ ranged between $10 \mathrm{~s}$ and $60 \mathrm{~s}$, depending on the air flow rate, while $t_{50}$ was comprised between $4 \mathrm{~min}$ and $60 \mathrm{~min}$. The ratio between $T$ and $t_{50}$ ranged between 10 and 180, thus validating the hypothesis.

$T=\frac{V}{Q_{v}}$ 
The concentration variation at the photocatalytic module inlet during $T$ was calculated. It enabled the inlet concentration at time $t-T$ to be considered the outlet concentration at time $t$ (Eq. (10)).

$r(t)=-\frac{C_{I N}(t)-C_{I N}(t-T)}{T}=\frac{C_{O U T}(t)-C_{I N}(t)}{T}$

The flow rate $Q_{V}\left(\mathrm{~N} \mathrm{~m}^{3} \cdot \mathrm{h}^{-1}\right)$ can be written as air velocity $v(\mathrm{~m}$. $\mathrm{s}^{-1}$ ) at the photocatalytic module inlet multiplied by the reactor cross-section $S=0.04 \mathrm{~m}^{2}$. For a given reaction time $t$, a relation between the reaction rate $r$, air velocity $v$ and the removal efficiency $R E$ was then deduced from Eqs. (6), (9) and (10). This relation is written below (Eq. (11)).

$r(t)=v \cdot \frac{S}{V} \cdot C(t) \cdot R E(t)$

From the experimental results, it could also be noticed that, in the exponential part of the curves, the ratio of reaction rate over concentration $(r / C)$ remained constant and equal to $k^{\prime}$. Consequently, for this part of the reaction curves, the removal efficiency does not depend on time either and can be written as follows (Eq. (12)).

$R E=\frac{1}{v} \cdot \frac{V}{S} \cdot k^{\prime}$

Next, Eq. (12) was used to calculate the RE values of each experiment. A statistical test was performed on $R E$ as done previously for the kinetic descriptors $r_{0}$ and $k$ '. The p-values are given in Table 2. This test shows that $R E$ significantly depends on both light irradiance $I$ and air velocity $v$. The $R E$ calculation from $k^{\prime}$ enabled us to study the role of velocity, by distinguishing the efficiency effects from the module feeding effect. It is a useful kinetic descriptor in order to write a reaction rate law as a function of the operating parameters.

\subsection{Writing the kinetic law}

According to the Langmuir-Hinshelwood model (Eq. (1)) and previous considerations, a kinetic law expressing the reaction rate as a function of the kinetic descriptors, $B$ and $R E$, and the concentration $C$ could be written (Eq. (13)).

$r(I, v, C)=\frac{C}{1+B \cdot C} \cdot \frac{S}{V} \cdot v \cdot R E(I, v)$

The next steps consisted of determining how the single-pass removal efficiency $R E$ depends on the operating parameters $I$ and $v$. The objective was to find a mathematical function, $R e=f(I, v)$, looking at both physical mechanisms and experimental results.

First of all, the effect of air velocity was investigated. This parameter is known to present antagonistic effects. For example, in a dynamic system, operating in a continuous mode, an increase in the air velocity improves the mass transfer $[26,27]$ but decreases the contact time, defined by the time of contact between the pollutant to be degraded and the photocatalytic medium. The contact time is proportional to the residence time when the same photocatalytic material is used. The residence time is itself proportional to the air velocity. Some authors have observed a positive effect of the increase in velocity, for flow rates ranging from 0.006 to $0.030 \mathrm{~m}^{3} \cdot \mathrm{h}^{-1}$ with a constant cross-section of $78.5 \mathrm{~cm}^{2}$, on the degradation efficiency [28]. This effect has been attributed to the improvement in mass transfer. In fact, if the dynamic regime is appropriate, i.e. for a Reynolds number over 300 [26], there is no mass transfer limitation. In the present work, the photocatalytic module was located in a $20 \mathrm{~cm} \times 20 \mathrm{~cm}$ square cross-section so that the hydraulic diameter $D_{H}$ was $20 \mathrm{~cm}$. The Reynolds number thus ranged between 2500 and 13,000, depending on the air velocity value $\left(0.2-1.0 \mathrm{~m} . \mathrm{s}^{-1}\right)$, and the reaction rate was not limited by mass transfer. The effect of the contact time on $R E$ had then to be investigated. In the literature, other studies have shown that when the velocity is raised the conversion yield is reduced, due to an insufficient contact time for flow rates between 0.02 and $1 \mathrm{~m}^{3}$. $\mathrm{h}^{-1}[11,27]$. Quici et al. [29], Zhong et al. [30] and Destaillats et al. [31] have observed that PCO cleaning efficiency is not improved when the air recirculation rate is set at higher values. Some authors have shown that for single-pass continuous flow reactors, the removal efficiency drops with the velocity [32,33] due to the decrease in the residence time $[29,34]$. The role of the contact time has been evidenced by changing both the flow rate and the reactor length [35]. In the present study, the contact length $l$ between the air and the photocatalyst was fixed but not known. For this reason, the inverse of air velocity $1 / v$ was used to assess the role of the contact time on the single-pass removal efficiency $R E$. In fact, the residence time $\tau$ is equal to the contact length $l$ divided by the air velocity $v$. In this work, $R E$ was calculated for each experiment in the exponential part of the curves using Eq. (12). In Fig. 7, $R E$ is plotted versus $1 / v$ for the three values of light irradiance $I$, while ignoring $C_{0}$ values.

According to Fig. 6, the removal efficiency increases with $1 / v$ and thus with the residence time. This result is in accordance with the literature as the relation between the removal efficiency and the residence time in single-pass reactors has been reported to be exponential (Eq. (14)) [36,37]. In this equation, $\tau=l / v$ is the residence time and $k_{r}$ is the reaction kinetic constant at the photocatalyst scale [36].

$R E=1-e^{-k_{r} \cdot \tau}=1-e^{-k_{r} \cdot \frac{1}{v}}$

The mathematical relation $R E=f(I, v)$ can then be expressed as in Eq. (15) below:

$R E=1-e^{\frac{-f(l)}{v}}$

To investigate the role of the light irradiance and thus to determine the function $f(I), R E$ was plotted versus $I$ (Fig. 8).

For each value of air velocity, the experimental curve could be modeled by a power law. This result is consistent with the literature as several authors have evidenced that the photocatalytic reaction kinetic constant $k_{r}$ depends on the light irradiance I with a power law [24,25]. $f(I)$ was thus modeled as a power law, which provided a model in which $R E$ depends simultaneously on both $I$ and $v$ as described in Eq. (16) where $a$ and $n$ have to be further determined.

$R E(I, v)=1-e^{\frac{-a, l^{n}}{v}}$

Thanks to Eqs. (12) and (16), the expression of the apparent first-order kinetic constant for the late part of the curves $k^{\prime}$ as a function of $I$ and $v$ can be written as in Eq. (17).

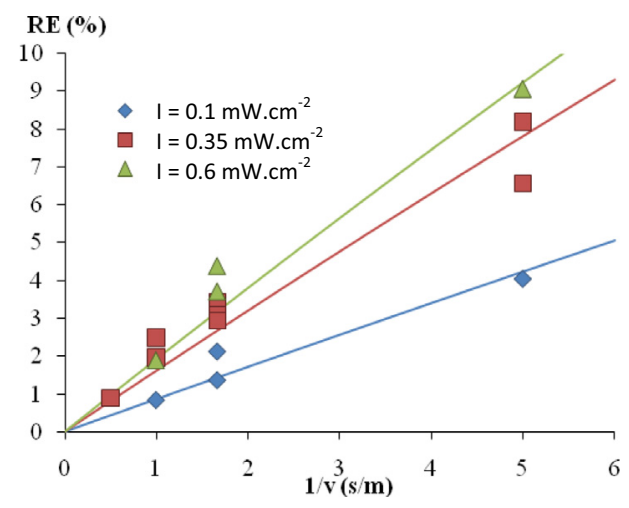

Fig. 7. Single-pass removal efficiency (RE) versus $1 / v$ for three values of light irradiance. 


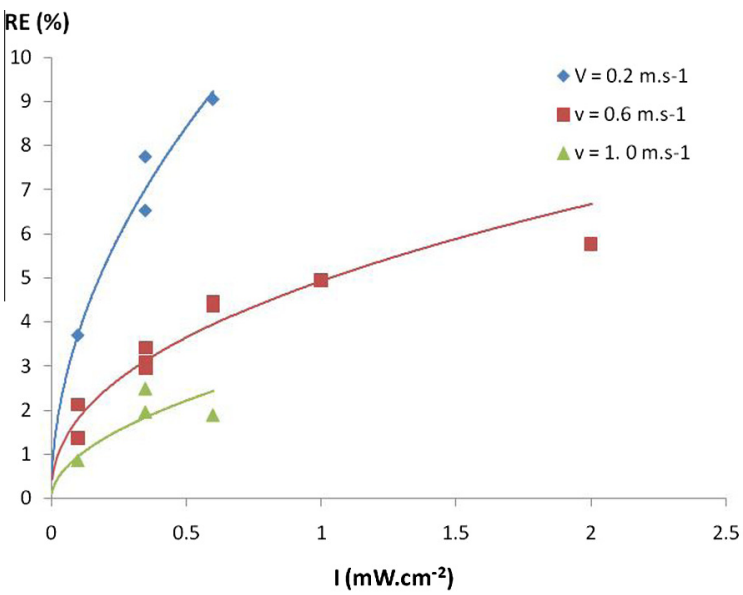

Fig. 8. Single-pass removal efficiency (RE) versus UV irradiance I for three values of air velocity.

Table 4

Obtained values of the kinetic rate law constants $a, n$ and $B$.

\begin{tabular}{ll}
\hline Constant & Value \\
\hline$\left.a\left(\mathrm{~m}^{-1}\right)^{-1}\right)$ & $0.0264 \pm 0.0011$ \\
$n$ & $0.471 \pm 0.045$ \\
$B\left(\mathrm{ppbv}^{-1}\right)$ & $0.0016 \pm 0.0004$
\end{tabular}

$K^{\prime}(I, v)=v \cdot \frac{S}{V} \cdot\left(1-e^{\frac{-a \cdot l^{n}}{v}}\right)$

Finally, a kinetic rate law that gives the reaction rate as a function of the concentration, the light irradiance and the air velocity can be written (Eq. (18)).

$r(I, v, C)=\frac{v \cdot \frac{S}{V} \cdot\left(1-e^{\frac{-a \cdot I^{n}}{v}}\right) \cdot C}{1+B \cdot C}$

This equation involves three constants $a, n$ and $B$ that have to be further determined. It can also be used to calculate the initial reaction rate $r_{0}$ as a function of $I, v$ and $C_{0}$.

\subsection{Determination of the kinetic rate law constants $a, n$ and $B$}

The values of the kinetic rate law constants $a, n$ and $B$ were determined by using the values of two different kinetic parameters obtained from the experimental results. The degradation curves can be almost completely described by $r_{0}$ for the beginning of the reaction and by $k^{\prime}$ for the end of the reaction. It was previously shown that $k^{\prime}$ can be replaced by $R E$. The calculated values of $R E$ were thus used to determine the constants $a$ and $n$, while the $r_{0}$ values were used to determine the constant $B$ using Eqs. (16) and (18) and Microsoft Excel Solver software. The values obtained for $a, n$ and $B$ are summarized in Table 4 .

To assess the quality of the adjustment for $a, n$ and $B$ values, two parity diagrams were plotted (Figs. 9 and 10). Fig. 9 shows the fitted values of $R E$ versus the calculated ones. Each point corresponds to an experiment. The points are well-distributed around the bisector line. Fig. 10 presents the fitted values of $r_{0}$ versus the calculated ones. This fit is not as good as that for the RE parameter. This is due to the error in the determination of $B$, which affects the $r_{0}$ calculation, especially for the experiments with the lowest initial concentrations.

It can also be noticed that $n$ is not significantly different from 0.5 (Table 4). This is consistent with the literature as it is generally considered that the $n$ exponent can take values between 0 and 1 .

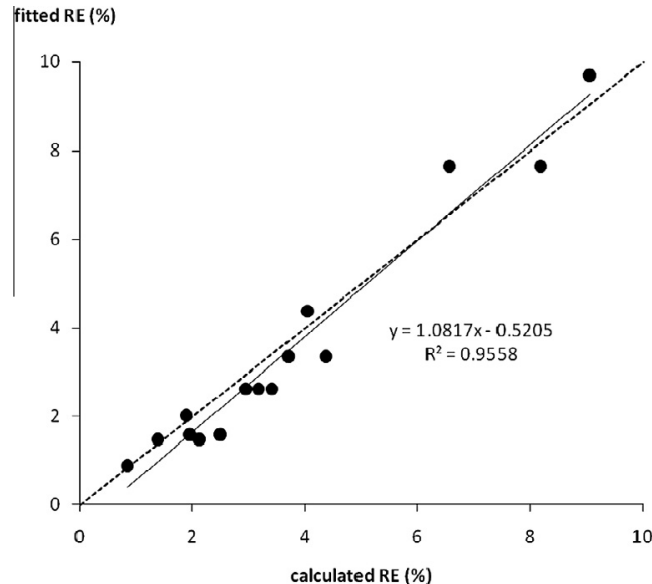

Fig. 9. Comparison between the fitted and calculated single-pass removal efficiencies. Experimental values (dots), linear fit (hard line) and $\mathrm{y}=\mathrm{x}$ bisector (dashed line).

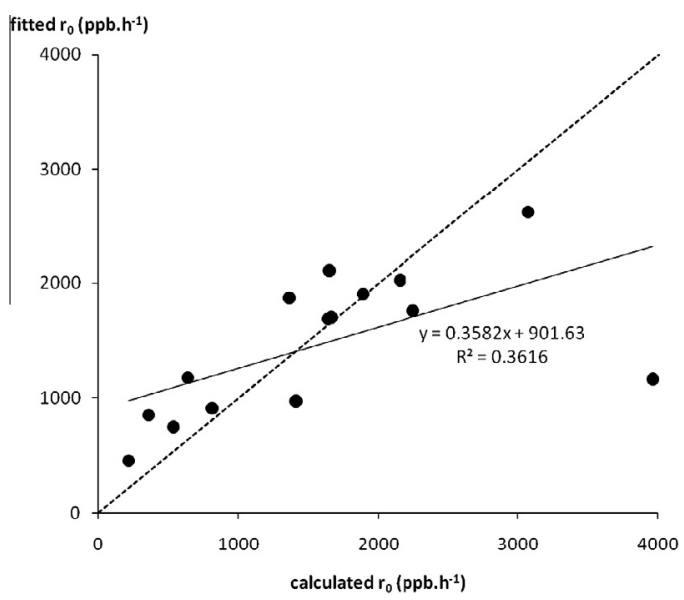

Fig. 10. Comparison between the fitted and calculated initial degradation rates. Experimental values (dots), linear fit (hard line) and $\mathrm{y}=\mathrm{x}$ bisector (dashed line).

Herrmann [38] reported that the influence of light irradiance on the reaction rate may be divided into three different regimes: (i) first order where the electron/hole pairs are consumed more rapidly by the chemical reaction than by the recombination phenomenon, (ii) 0.5 order where the recombination rate is predominant and (iii) 0 order. According to Modestov and Lev [39], the $n$ exponent can also change from 1 to 0.5 over a certain level of photonic flux due to an excess of photogenerated species $\left(\mathrm{e}^{-}, \mathrm{h}^{+}\right.$and $\mathrm{OH}^{\circ}$ ). In a previous study, Raillard et al. [8] found values for $n$ ranging from 0.59 to 0.69 and concluded that the regime driven by light irradiance was principally governed by a recombination phenomenon under UV A. The same conclusion can be made in the present work.

\subsection{Kinetic rate law validation}

Four complementary experiments were performed to validate the kinetic rate law. One was carried out with $I, v$ and $C_{0}$ values chosen in the range of the design of experiments. It enabled the law to be assessed in the condition range for which this law was written (Fig. 4). Three other experiments were carried out for higher values of $I, v$ or $C_{0}$. The aim of these degradations was to assess if this law can predict the degradation kinetics of toluene outside the range in which the law was written. The conditions of the four validation degradations are given in Table 5 . 

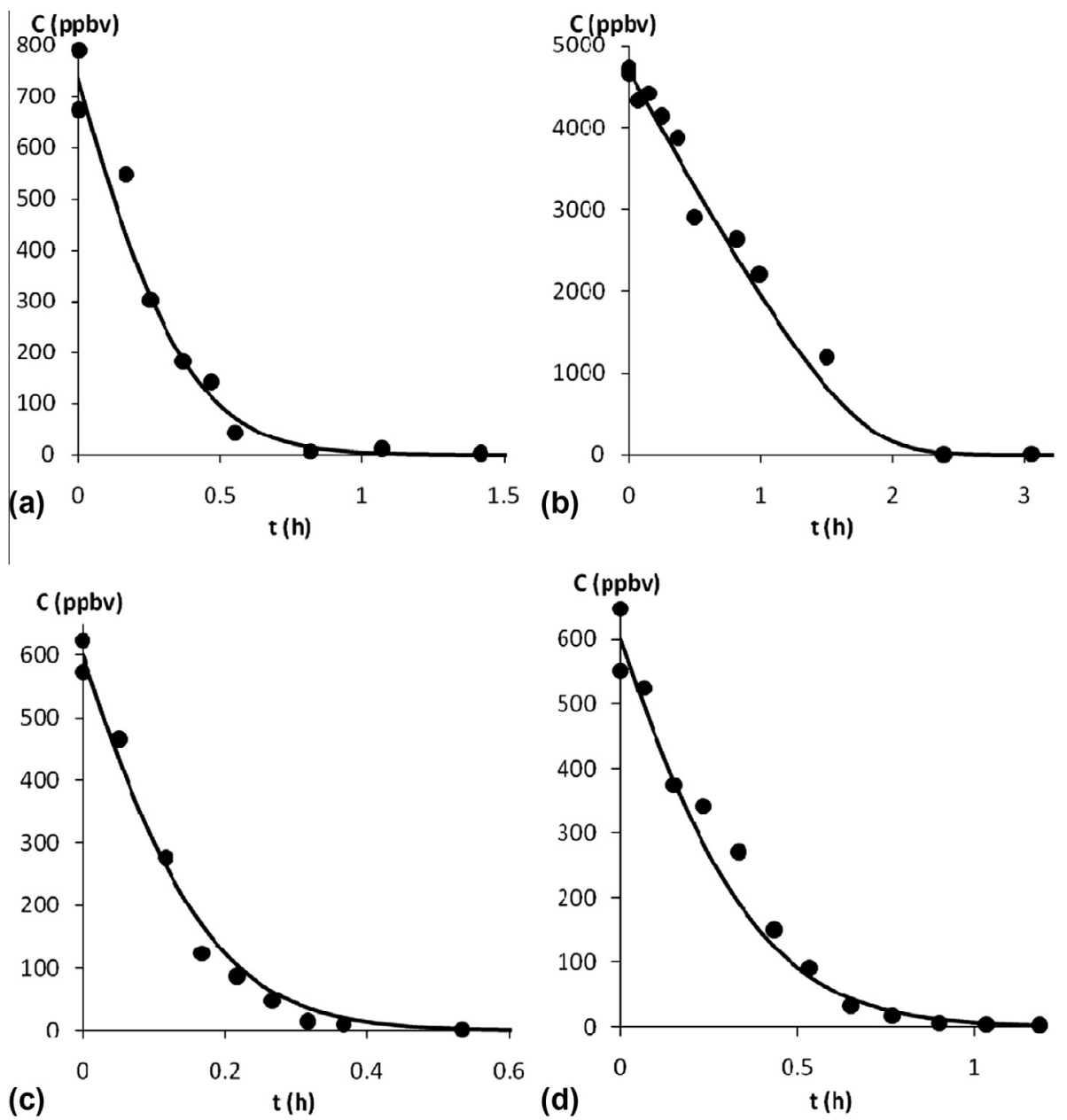

Fig. 11. Validation experiments. Experimental values (dots) and simulated degradation with the kinetic law (lines). Experimental conditions are given in Table 5.

Table 5

Experimental conditions for the kinetic law validation.

\begin{tabular}{lllll}
\hline Experiment & $I\left(\mathrm{~mW} . \mathrm{cm}^{-2}\right)$ & $v\left({\left.\mathrm{~m} . \mathrm{s}^{-1}\right)}^{-}\right.$ & $C_{0}(\mathrm{ppbv})$ & Reference in Fig. 11 \\
\hline In cube & 0.50 & 0.2 & 732 & $\mathrm{a}$ \\
$\mathrm{I}^{+}$ & 2.00 & 0.6 & 599 & $\mathrm{c}$ \\
$\mathrm{v}^{+}$ & 0.35 & 1.9 & 599 & $\mathrm{~d}$ \\
$\mathrm{C}_{0}^{+}$ & 0.35 & 0.6 & 4694 & $\mathrm{~b}$ \\
\hline
\end{tabular}

Fig. 11 illustrates the comparison between the experimental results and the curves plotted using the kinetic law for four experimental conditions. These curves indicate that the established kinetic model fits properly the experimental data even for experimental conditions different from those defined in the design matrix ( $R^{2}$ comprised between 0.97 and 0.99 for the 4 experiments). This result validates the methodology used in this work, i.e. the establishment of a kinetic rate law using both an empirical and a physico-chemical approach. The fact that this law succeeded in being predictive for conditions outside of the experimental design range is thought to be due to its semi-empirical nature. A fully empirical law would not be as efficient for these conditions.

\section{Conclusion}

Specific concerns about PCO performance for indoor air quality improvement are associated with understanding the role of operating parameters in environmental conditions close to real ones.
In this work, the influence of three operating parameters on the photocatalytic oxidation of toluene in a closed-loop multi-pass photocatalytic reactor was investigated. The chosen operating parameters were initial toluene concentration, light irradiance and air flow velocity. A design of experiments was used to carry out this three-parameter study. The objective of this work was to propose a kinetic model valid for specific indoor air conditions (humid air and sub-ppmv concentrations) and based on both experimental results and theoretical considerations. Our results highlight that:

i. The statistical analysis of the results obtained from the DOE is not sufficient to understand the role of all the operating parameters in the reaction rate. In particular, this approach does not allow the influence of the stream velocity $v$ to be explored.

ii. The calculation of the single-pass removal efficiency $R E$ can be used to distinguish mass flow rate effects from the intrinsic efficiency of the photocatalytic module. $R E$ was found to depend significantly on both light irradiance and stream velocity. It is a useful kinetic descriptor for writing a reaction rate law as a function of the operating parameters.

A relationship between the single-pass removal efficiency, light irradiance and stream velocity was then established and introduced into the expression of the overall reaction rate, the latter having been written according to the Langmuir-Hinshelwood model. It was shown that the model fits properly the degradation 
curves for experimental conditions defined in the DOE and for other experimentations. A similar work can be conducted with other VOCs. The influence of the parameters will follow the same trend but the constants $a$ and $B$ would have to be recalculated. In a future work, the kinetic rate law will also be tested on the degradation of mixtures of VOCs at ppb range concentrations.

\section{References}

[1] P. Wolkoff, G.D. Nielsen, Organic compounds in indoor air-their relevance for perceived indoor air quality?, Atmos Environ. 35 (2001) 4407-4417.

[2] M. Hodgson, H. Levin, P. Wolkoff, Volatile organic compounds and indoor air, J. Allergy Clin. Immunol. 94 (1994) 296-303.

[3] C. Billionnet, E. Gay, S. Kirchner, B. Leynaert, I. Annesi-Maesano, Quantitative assessments of indoor air pollution and respiratory health in a populationbased sample of French dwellings, Environ. Res. 111 (2011) 425-434.

[4] A. Norhidayah, L. Chia-Kuang, M.K. Azhar, S. Nurulwahida, Indoor air quality and sick building syndrome in three selected buildings, Procedia Eng. 53 (2013) 93-98.

[5] F. Squinazi, La pollution de l'air à l'intérieur des bâtiments (allérgènes exclus), Rev. Fr. Allergol. Immunol. Cli. 42 (2002) 248-255.

[6] C. Raillard, V. Héquet, P. Le Cloirec, J. Legrand, Kinetic study of ketones photocatalytic oxidation in gas phase using $\mathrm{TiO}_{2}$ containing paper: effect of water vapor, J. Photochem. Photobiol. Chem. 163 (2004) 425-431.

[7] O. Debono, F. Thévenet, P. Gravejat, V. Héquet, C. Raillard, L. Le Coq, N. Locoge Gas phase oxidation of decane at ppb levels: removal kinetics, reaction intermediates and carbon mass balance, J. Photochem. Photobiol. Chem. 258 (2013) 17-29.

[8] C.L. Bianchi, S. Gatto, C. Pirola, A. Naldoni, A. Di Michele, G. Cerrato, V. Crocellà, V. Capucci, Photocatalytic degradation of acetone, acetaldehyde and toluene in gas phase: comparison between nano and micro-sized $\mathrm{TiO}_{2}$, Appl. Catal. B Environ. 146 (2014) 123-130.

[9] D.F. Ollis, Photocatalytic purification and remediation of contaminated air and water, Comptes Rendus Académie Sci. Ser. IIC Chem. 3 (2000) 405-411.

[10] N.N. Lichtin, M. Avudaithai, E. Berman, A. Grayfer, TiP2-photocatalyzed oxidative degradation of binary mixtures of vaporized organic compounds, Sol. Energy 56 (1996) 377-385.

[11] M. Sleiman, P. Conchon, C. Ferronato, J.-M. Chovelon, Photocatalytic oxidation of toluene at indoor air levels (ppbv): towards a better assessment of conversion, reaction intermediates and mineralization, Appl. Catal. B Environ. 86 (2009) 159-165.

[12] D. Vildozo, R. Portela, C. Ferronato, J.-M. Chovelon, Photocatalytic oxidation of 2-propanol/toluene binary mixtures at indoor air concentration levels, Appl. Catal. B Environ. 107 (2011) 347-354.

[13] Y. Paz, Application of $\mathrm{TiO}_{2}$ photocatalysis for air treatment: patents' overview, $\mathrm{TiO}_{2}$ Photocatal. 25 Years 99 (2010) 448-460.

[14] N. Costarramone, B. Kartheuser, C. Pecheyra, T. Pigot, S. Lacombe, Efficiency and harmfulness of air-purifying photocatalytic commercial devices: from standardized chamber tests to nanoparticles release, Catal. Today, 8th European Meeting on Solar Chemistry and Photocatalysis: Environmental Applications $252 \quad$ (2015) 35-40, http://dx.doi.org/10.1016 j.cattod.2015.01.008.

[15] M.L. Sauer, D.F. Ollis, Acetone oxidation in a photocatalytic monolith reactor, J Catal. 149 (1994) 81-91.

[16] W. Choi, J.Y. Ko, H. Park, J.S. Chung, Investigation on $\mathrm{TiO}_{2}$-coated optical fibers for gas-phase photocatalytic oxidation of acetone, Appl. Catal. B Environ. 31 (2001) 209-220.

[17] Y. Zhang, R. Yang, R. Zhao, A model for analyzing the performance of photocatalytic air cleaner in removing volatile organic compounds, Atmos. Environ. 37 (2003) 3395-3399.
[18] G. Li Puma, I. Salvadó-Estivill, T.N. Obee, S.O. Hay, Kinetics rate model of the photocatalytic oxidation of trichloroethylene in air over $\mathrm{TiO}_{2}$ thin films, Sep. Purif. Technol. 67 (2009) 226-232.

[19] S.W. Verbruggen, S. Lenaerts, S. Denys, Analytic versus CFD approach of kinetic modeling of gas phase photocatalysis, Chem. Eng. J. 262 (2015) 1-8.

[20] C. Raillard, A. Maudhuit, V. Héquet, L. Le Coq, J. Sablayrolles, L. Molins, Use of experimental designs to establish a kinetic law for a gas phase photocatalytic process, Int. J. Chem. React. Eng. 12 (2014) 113-122.

[21] A. Maudhuit, C. Raillard, V. Héquet, L. Le Coq, J. Sablayrolles, L. Molins, Adsorption phenomena in photocatalytic reactions: the case of toluene, acetone and heptane, Environ. Nanotechnol. 170 (2011) 464-470.

[22] J. Taranto, D. Frochot, P. Pichat, Modeling and optimizing irradiance on planar, folded, and honeycomb shapes to maximize photocatalytic air purification, Mater. Appl. Process. Photocatal. 122 (2007) 66-77.

[23] G. Box, D. Behnken, Some new three level designs for the study of quantitative variables, Technometrics 2 (1960) 455-475.

[24] J. Mo, Y. Zhang, Q. Xu, J.J. Lamson, R. Zhao, Photocatalytic purification of volatile organic compounds in indoor air: a literature review, Atmos. Environ. 43 (2009) 2229-2246.

[25] A.A. Assadi, A. Bouzaza, D. Wolbert, Photocatalytic oxidation of trimethylamine and isovaleraldehyde in an annular reactor: influence of the mass transfer and the relative humidity, J. Photochem. Photobiol. Chem. 236 (2012) 61-69.

[26] T.N. Obee, Photooxidation of sub-parts-per-million toluene and formaldehyde levels on titania using a glass-plate reactor, Environ. Sci. Technol. 30 (1996) 3578-3584.

[27] W.-K. Jo, S.-H. Shin, Photocatalytic decomposition of mobile-source related pollutants using a continuous-flow reactor, J. Environ. Sci. 22 (2010) 460-466.

[28] K.-H. Wang . H.-H. Tsai, Y.-H. Hsieh, The kinetics of photocatalytic degradation of trichloroethylene in gas phase over $\mathrm{TiO}_{2}$ supported on glass bead, Appl. Catal. B Environ. 17 (1998) 313-320.

[29] N. Quici, M.L. Vera, H. Choi, G.L. Puma, D.D. Dionysiou, M.I. Litter, H. Destaillats, Effect of key parameters on the photocatalytic oxidation of toluene at low concentrations in air under $254+185 \mathrm{~nm}$ UV irradiation, Appl. Catal. B Environ. 95 (2010) 312-319.

[30] L. Zhong, F. Haghighat, P. Blondeau, J. Kozinski, Modeling and physical interpretation of photocatalytic oxidation efficiency in indoor air applications, Build. Environ. 45 (2010) 2689-2697.

[31] H. Destaillats, M. Sleiman, D.P. Sullivan, C. Jacquiod, J. Sablayrolles, L. Molins, Key parameters influencing the performance of photocatalytic oxidation (PCO) air purification under realistic indoor conditions, Appl. Catal. B Environ. 128 (2012) 159-170.

[32] M. Sung, S. Kato, F. Kawanami, M. Sudo, Evaluation of an air-cleaning unit having photocatalytic sheets to remove acetaldehyde from indoor air, Build. Environ. 2009 (45) (2010) 2002-2007.

[33] J. Lyu, L. Zhu, C. Burda, Considerations to improve adsorption and photocatalysis of low concentration air pollutants on $\mathrm{TiO}_{2}$, Catal. Today 225 (2014) 24-33.

[34] W. Choi, J.Y. Ko, H. Park, J.S. Chung, Investigation on $\mathrm{TiO}_{2}$-coated optical fibers for gas-phase photocatalytic oxidation of acetone, Appl. Catal. B Environ. 31 (2001) 209-220.

[35] J.-M. Herrmann, Heterogeneous photocatalysis: an emerging discipline involving multiphase systems, Catal. Multiphase React. 24 (1995) 157-164.

[36] A.T. Hodgson, H. Destaillats, D.P. Sullivan, W.J. Fisk, Performance of ultra-violet photocatalytic oxidation for indoor air cleaning applications, Indoor Air 17 (2007) 305-316.

[37] C.H. Ao, S.C. Lee, Enhancement effect of $\mathrm{TiO}_{2}$ immobilized on activated carbon filter for the photodegradation of pollutants at typical indoor air level, Appl. Catal. B Environ. 44 (2003) 191-205.

[38] J.-M. Herrmann, Heterogeneous catalysis: state of the art and present applications, Top. Catal. 34 (2005) 49-65.

[39] A. Modestov, O. Lev, Photocatalytic oxidation of 2,4-dichlorophenoxyacetic acid with titania photocatalyst. Comparison of supported and suspended $\mathrm{TiO}_{2}$, J. Photochem. Photobiol. A 112 (1998) 261-270. 